PROCEEDINGS OF THE

AMERICAN MATHEMATICAL SOCIETY

Volume 139, Number 9, September 2011, Pages 3181-3190

S 0002-9939(2011)10749-4

Article electronically published on February 3, 2011

\title{
GEOMETRY OF THE SIEGEL MODULAR THREEFOLD WITH PARAMODULAR LEVEL STRUCTURE
}

\author{
CHIA-FU YU
}

(Communicated by Lev Borisov)

\begin{abstract}
In this paper we extend some results of Norman and Oort and of de Jong, and give an explicit description of the geometry of the Siegel modular threefold with paramodular level structure. We also discuss advantages and restrictions of three standard methods for studying moduli spaces of abelian varieties.
\end{abstract}

\section{INTRODUCTION}

In this paper we study the arithmetic model of the Siegel modular three-fold with paramodular level structure at a prime $p$. This is a very special case of geometric objects considered in the works of Norman and Oort [10] and of de Jong [2]. We extend their work and determine the singularities in this case. Our result can be used for computing the cohomology groups of the Siegel 3-fold by the PicardLefschetz formula (see 4, Expose XV).

Let $p$ be a rational prime number, $N \geq 3$ a prime-to- $p$ positive integer. We choose a primitive $N$ th root of unity $\zeta_{N}$ in $\overline{\mathbb{Q}} \subset \mathbb{C}$ and an embedding $\overline{\mathbb{Q}} \hookrightarrow \overline{\mathbb{Q}}_{p}$. Let $\mathbf{A}_{2, p, N}$ denote the moduli scheme over $\mathbb{Z}_{(p)}\left[\zeta_{N}\right]$ of polarized abelian surfaces $(A, \lambda, \eta)$ with polarization degree $\operatorname{deg} \lambda=p^{2}$ and with a symplectic level- $N$ structure with respect to $\zeta_{N}$. We denote by $\mathcal{A}_{2, p, N}:=\mathbf{A}_{2, p, N} \otimes \overline{\mathbb{F}}_{p}$ the reduction modulo $p$ of the moduli scheme $\mathbf{A}_{2, p, N}$.

In 10] Norman and Oort study the p-rank stratification on Siegel moduli spaces (the case for principally polarized abelian varieties was studied earlier in Koblitz [7]), which we recall as follows. An abelian variety over a field $K$ of characteristic $p$ is said to have $p$-rank $f$ if $\operatorname{dim}_{\mathbb{F}_{p}} A[p](\bar{K})=f$, which is an integer between zero and $\operatorname{dim} A$. Conversely, given any integer $0 \leq f \leq g$, there is a $g$-dimensional abelian variety with $p$-rank $f$. The discrete invariant $p$-rank defines locally closed subsets in the moduli space; it is showed in [10] that these subsets form a stratification. We now focus on the Siegel 3-fold. For each integer $0 \leq f \leq 2$, let $V_{f} \subset \mathcal{A}_{2, p, N}$ (resp. $\left.V_{\leq f} \subset \mathcal{A}_{2, p, N}\right)$ be the reduced subscheme consisting of points with $p$-rank equal to $f$ (resp. equal to or less than $f$ ). The ordinary locus, which parametrizes the ordinary abelian varieties, is the $p$-rank 2 stratum; the non-ordinary locus is $V_{\leq 1}$.

Received by the editors January 23, 2010 and, in revised form, August 11, 2010 and August 19, 2010.

2010 Mathematics Subject Classification. Primary 14G35, 14K10, 11G18, 32S25.

Key words and phrases. Siegel modular varieties, local models, paramodular level structure.

(C)2011 American Mathematical Society Reverts to public domain 28 years from publication 
We have the following fundamental results for $\mathbf{A}_{2, p, N}$ (see [11, Theorem 2.3.3; [10, Theorems 3.1 and 4.1; 2, Theorem 1.12 and Proposition 3.3):

Theorem 1.1. (1) The structure morphism $\mathbf{A}_{2, p, N} \rightarrow \operatorname{Spec} \mathbb{Z}_{(p)}\left[\zeta_{N}\right]$ is flat and a complete intersection morphism of relative dimension 3 . The fibers are geometrically irreducible.

(2) The stratum $V_{f}$ is dense in $V_{<f}$ and $\operatorname{dim} V_{f}=1+f$ for $f=0,1,2$.

(3) The non-ordinary locus $V_{\leq 1}$ has two irreducible components.

Let $\mathcal{S}_{2, p, N} \subset \mathcal{A}_{2, p, N}$ denote the supersingular locus, which is the reduced closed subscheme over $\overline{\mathbb{F}}_{p}$ consisting of supersingular abelian varieties. Recall that an abelian variety $A$ in characteristic $p$ is called supersingular if it is isogenous to a product of supersingular elliptic curves over an algebraically closed field $k$; it is called superspecial if it is isomorphic to a product of supersingular elliptic curves over $k$. Since any abelian surface of $p$-rank zero is supersingular, the supersingular locus $\mathcal{S}_{2, p, N}$ is the same as $V_{0}$. Let $\Lambda_{2,1, N} \subset \mathcal{A}_{2,1, N}\left(\overline{\mathbb{F}}_{p}\right)$ be the set of superspecial points in the moduli space $\mathcal{A}_{2,1, N}$ of principally polarized abelian surfaces with level- $N$ structure. Let $\Lambda \subset \mathcal{S}_{2, p, N}\left(\overline{\mathbb{F}}_{p}\right)$ be the subset consisting of superspecial points $(A, \lambda, \eta)$ such that $\operatorname{ker} \lambda \simeq \alpha_{p} \times \alpha_{p}$. The following is a description of the supersingular locus (see [15, Theorem 4.7):

Theorem 1.2. (1) The scheme $\mathcal{S}_{2, p, N}$ is equi-dimensional, and each irreducible component is isomorphic to $\mathbf{P}^{1}$ over $\overline{\mathbb{F}}_{p}$.

(2) The scheme $\mathcal{S}_{2, p, N}$ has $\left|\Lambda_{2,1, N}\right|$ irreducible components.

(3) The singular locus of $\mathcal{S}_{2, p, N}$ is the subset $\Lambda$. Moreover, at each singular point there are $p^{2}+1$ irreducible components passing through and any two of them intersect transversely.

See [15], Corollary 3.3 and Theorem 4.1, for a precise formula for $\left|\Lambda_{2,1, N}\right|$ and $|\Lambda|$, respectively.

In this paper we prove the following:

Theorem 1.3. The moduli scheme $\mathbf{A}_{2, p, N}$ is regular and smooth over $\mathrm{Spec} \mathbb{Z}_{(p)}\left[\zeta_{N}\right]$ exactly away from the subset $\Lambda$. At each singular point $x$ (in $\Lambda$ ) in the special fiber $\mathcal{A}_{2,1, N}$, the formal completion of the local ring at $x$ is isomorphic to

$$
W\left(\overline{\mathbb{F}}_{p}\right)\left[\left[z_{11}, z_{12}, z_{21}, z_{22}\right]\right] /\left(p+z_{11} z_{22}-z_{12} z_{21}\right),
$$

where $W\left(\overline{\mathbb{F}}_{p}\right)$ is the ring of Witt vectors over $\overline{\mathbb{F}}_{p}$. Consequently, the moduli space $\mathcal{A}_{2, p, N}$ is normal.

Theorem 1.3 improves Theorem 1.1 (1). Theorems 1.11 .3 provide a good understanding of the geometry of the moduli scheme $\mathbf{A}_{2, p . N}$. As the singularities are non-degenerate ordinary double points, one can use the Picard-Lefschetz formula to compute the cohomology groups of the moduli space $\mathbf{A}_{2, p, N}$. We intend to continue the work along this direction.

The paper is organized as follows. In Section 2 we study the singularity of the moduli scheme using the crystalline theory when $p>2$. We also discuss the classification of deformations of non-degenerate double points. In Section 3 we determine the singularity of the moduli scheme using the local model for arbitrary residue characteristic. In Section 4 we explain that some higher terms of the defining equation in Norman's first example in [9] are required in order to describe the nonordinary locus. 
Notation. For any $\operatorname{ring} R$, any $R$-module $M$ and any subset $S$ of $M$, we denote by $\langle S\rangle_{R}$, or simply $\langle S\rangle$ as long as the ground ring $R$ is understood, the $R$-submodule generated by $S$.

\section{Singularities USING THE CRYSTAlline theory}

In this section we use the crystalline theory to determine the singularities of the moduli scheme $\mathbf{A}_{2, p . N}$ for $p>2$. Standard references for the Grothendieck-Messing deformation theory are [6] and [8].

2.1. The smooth locus of $\mathbf{A}_{2, p, N}$. Let $k$ be an algebraically closed field of characteristic $p$. Let $W:=W(k)$ be the ring of Witt vectors over $k$, and let $B(k)$ be the fraction field of $W(k)$. Let $\sigma$ be the Frobenius map on $W$ and $B(k)$, respectively. In this paper we use the covariant Dieudonné theory. Let $A$ be an abelian variety over $k$. Denote by $M(A)$ the (covariant) Dieudonné module of $A$, while the classical Dieudonné functor is denoted by $M^{*}$. The Dieudonné module $M(A)$ is canonically isomorphic to the dual $\left[M^{*}(A)\right]^{t}$ of the classical Dieudonné module $M^{*}(A)$ of $A$. We recall that for any Dieudonné module $M$, the dual Dieudonné module $M^{t}$ of $M$ is defined as $\operatorname{Hom}_{W}(M, W(1))$ together with natural operations by $F$ and $V$. If $M$ is the Dieudonné module of an abelian variety $A$, then $M^{t}$ is naturally isomorphic to the Dieudonné module of the dual abelian variety $A^{t}$. If $M$ is equipped with a quasi-polarization, then it is convenient to identify $M^{t}$ with the dual lattice of $M$ in $M_{B(k)}=M \otimes_{W} B(k)$ with respect to the alternating pairing. The Dieudonné modules considered here are finite and free as $W$-modules.

For convenience of discussion, we introduce the following definition.

Definition 2.1. A point $(A, \lambda, \eta)$ in $\mathbf{A}_{2, p, N}(k)$ is called Lagrangian if there exists a $W$-basis $X_{1}, X_{2}, Y_{1}, Y_{2}$ for $M=M(A)$ such that $Y_{1}, Y_{2} \in V M$,

$$
\left\langle X_{1}, Y_{1}\right\rangle=-\left\langle Y_{1}, X_{1}\right\rangle=1, \quad\left\langle X_{2}, Y_{2}\right\rangle=-\left\langle Y_{2}, X_{2}\right\rangle=p,
$$

and the other pairings are zero.

Remark 2.2. An equivalent definition (for general Siegel moduli spaces) is that a quasi-polarized Dieudonné module $M$ is called Lagrangian if there is a maximally isotropic $W$-sublattice $L \subset M$ such that $L$ is co-torsion free and contained in $V M$. This is also equivalent to the condition that the corresponding polarized abelian variety can be lifted over $W$ (cf. [14).

Lemma 2.3. If $x=(A, \lambda, \eta) \in \mathbf{A}_{2, p, N}(k)$ is a Lagrangian point, then the moduli space $\mathbf{A}_{2, p, N}$ is smooth at $x$.

Proof. We choose a $W$-basis $X_{1}, X_{2}, Y_{1}, Y_{2}$ for $M=M(A)$ as in Definition 2.1. We compute the Hodge filtration Fil of $H_{1}^{\mathrm{DR}}(A)=M / p M$ :

$$
\text { Fil }=V M / p M=<Y_{1}, Y_{2}>_{k} .
$$

Let $R$ be the first order deformation ring of $(M,\langle\rangle$,$) . The first order universal$ deformation of $M$ over $R$, by the Grothendieck-Messing deformation theory, is given by $\widetilde{\text { Fil }}=<\widetilde{Y}_{1}, \widetilde{Y}_{2}>_{R}$, where

$$
\tilde{Y}_{1}=Y_{1}+t_{11} X_{1}+t_{12} X_{2}, \quad \tilde{Y}_{2}=Y_{2}+t_{21} X_{1}+t_{22} X_{2},
$$

and $R$ is a quotient of

$$
W(k)\left[\left[t_{11}, t_{12}, t_{21}, t_{22}\right]\right] / \mathfrak{m}^{2}, \quad \mathfrak{m}:=\left(p, t_{11}, t_{12}, t_{21}, t_{22}\right),
$$


the first order deformation ring of $M$ (without polarization). One computes $\left\langle\widetilde{Y}_{1}, \widetilde{Y}_{2}\right\rangle=-t_{21}+p t_{12}$. This shows that

$$
R=W(k)\left[\left[t_{11}, t_{12}, t_{21}, t_{22}\right]\right] /\left(\left(-t_{21}+p t_{12}\right)+\mathfrak{m}^{2}\right),
$$

and hence the smoothness.

At least in the equi-characteristic situation, Lemma 2.3 is a special case of [1], Theorem 3.2.2.

Recall that the a-number of a Dieudonné module $M$ over $k$, which is denoted by $a(M)$, is defined to be $\operatorname{dim}_{k} M /(F, V) M$. Similarly, define $a(A):=a(M(A))$ to be the $a$-number of an abelian variety $A$.

Let $x=(A, \lambda, \eta)$ be a point in $\mathbf{A}_{2, p, N}(k)$ and let $M$ be the associated quasipolarized Dieudonné module. We have the following cases:

(i) $\mathbf{a}(\mathbf{A}) \leq \mathbf{1}$. It follows from [10, Theorem 2.3.3 that $x$ is a Lagrangian point.

(ii) $\mathbf{a}(\mathbf{A})=\mathbf{2}$. In this case $A$ is superspecial. It is not hard to classify such Dieudonné modules. There are two possibilities (notice that $k$ is algebraically closed):

(iia) There is a $W$-basis $X_{1}, X_{2}, Y_{1}, Y_{2}$ for $M$ with the following properties:

$$
\begin{gathered}
F X_{1}=Y_{1}, F Y_{1}=-p X_{1}, F X_{2}=Y_{2}, F Y_{2}=-p X_{2}, \\
\left\langle X_{1}, Y_{1}\right\rangle=-\left\langle Y_{1}, X_{1}\right\rangle=1, \quad\left\langle X_{2}, Y_{2}\right\rangle=-\left\langle Y_{2}, X_{2}\right\rangle=p,
\end{gathered}
$$

and the remaining pairings are zero.

(iib) There is a $W$-basis $X_{1}, X_{2}, Y_{1}, Y_{2}$ for $M$ with the following properties:

$$
\begin{gathered}
F X_{1}=Y_{1}, F Y_{1}=p X_{1}, F X_{2}=Y_{2}, F Y_{2}=p X_{2}, \\
\left\langle X_{1}, X_{2}\right\rangle=-\left\langle X_{2}, X_{1}\right\rangle=1, \quad\left\langle Y_{1}, Y_{2}\right\rangle=-\left\langle Y_{2}, Y_{1}\right\rangle=p,
\end{gathered}
$$

and the remaining pairings are zero.

Lemma 2.4. A point $x=(A, \lambda, \eta)$ of $\mathbf{A}_{2, p, N}(k)$ lies in the case (iib) above if and only if it lies in the finite set $\Lambda$.

Proof. Recall that if $H$ is the kernel of an isogeny $\varphi: A \rightarrow B$, then the Dieudonné module $M(B) / M(A)$ is isomorphic to $M^{*}\left(H^{D}\right)$, where $H^{D}$ is the Cartier dual of $H$. One only needs to consider the case (ii) as the $a$-number is equal to 2 for any member in $\Lambda$. In the case (iia), one has

$$
M^{t}=<X_{1}, Y_{1}, \frac{1}{p} X_{2}, \frac{1}{p} Y_{2}>\text {. }
$$

We have $F M^{t} \not \subset M$ and $V M^{t} \not \subset M$. Therefore, $\operatorname{ker} \lambda \nsucceq \alpha_{p} \times \alpha_{p}$.

In the case (iib), one has

$$
M^{t}=<X_{1}, X_{2}, \frac{1}{p} Y_{1}, \frac{1}{p} Y_{2}>\text {. }
$$

We have $F M^{t} \subset M$ and $V M^{t} \subset M$. Therefore, $\operatorname{ker} \lambda \simeq \alpha_{p} \times \alpha_{p}$. This proves the lemma.

Note that the point $x$ in the case (iia) is Lagrangian. We will show (Lemma 2.10) that the moduli space has singularities at points in the case (iib). It follows from Lemma 2.2 that the point $x$ in the case (iib) is not Lagrangian.

From the discussions above, we have proven 
Proposition 2.5. The smooth locus of the structure morphism $\mathbf{A}_{2, p, N} \rightarrow$ $\operatorname{Spec}_{(p)}\left[\zeta_{N}\right]$ is the complement of the finite subset $\Lambda$.

2.2. Classification of non-degenerate double points. Recall that a quadratic form $Q(x)$ over a local ring $A$ is called non-degenerate if the associated bilinear form

$$
B(x, y):=Q(x+y)-Q(x)-Q(y)
$$

is a perfect pairing. We show the following:

Proposition 2.6. Let $A$ be a complete Noetherian local ring with separably closed residue field $k$, and let $\mathfrak{m}_{A}$ be its maximal ideal. Let $R:=A\left[\left[x_{1}, \ldots, x_{n}\right]\right]$ be the ring of formal power series over $A, \mathfrak{m}_{R}$ its maximal ideal, and let $f(x) \in R$. Suppose

$$
f(x) \equiv a+Q(x) \quad \bmod \mathfrak{m}_{R}^{3},
$$

where $a$ is an element in $\mathfrak{m}_{A}$ and $Q(x)$ is a non-degenerate quadratic form. Then there is an isomorphism

$$
R /(f(x)) \simeq R /\left(a^{\prime} u+Q^{\prime}(x)\right)
$$

where $Q^{\prime}(x)$ is any non-degenerate quadratic form over $A, a^{\prime}$ is an element in $\mathfrak{m}_{A}$ such that $a^{\prime} \equiv a \bmod \mathfrak{m}_{A}^{3}$, and $u$ is any unit in $A$.

Since $A$ is strictly Henselian, any two non-degenerate quadratic forms are equivalent. We may take $Q^{\prime}(x)$ to be the standard split form. By rescaling half of the variables by suitable units in $A$, one shows that

$$
R /\left(a^{\prime} u+Q^{\prime}(x)\right) \simeq R /\left(a^{\prime}+Q^{\prime}(x)\right) .
$$

Lemma 2.7. Let $R:=A\left[\left[x_{1}, \ldots, x_{n}\right]\right]$ be the ring of formal power series over a local ring $A$. Let $I$ be the ideal of $R$ generated by $x_{1}, \ldots, x_{n}$ and $f(x)$ be an element of $R$. Suppose that

$$
f(x) \equiv a+Q(x) \quad \bmod I^{3},
$$

where $Q(x)$ is a non-degenerate quadratic form and $a \in \mathfrak{m}_{A}$. Then there is an isomorphism

$$
R /(f(x)) \simeq R /(a+Q(x)) .
$$

Proof. This is [5], Chapter III, Proposition 2.3.

Lemma 2.8. Let $A$ be a complete Noetherian local ring and $\mathfrak{m}_{A}$ be its maximal ideal. Let $R, \mathfrak{m}_{R}, I$, and $f(x)$ be as before. Suppose that

$$
f(x) \equiv a+\sum_{i=1}^{n} a_{i} x_{i}+Q(x) \bmod I^{3},
$$

where $a, a_{i}$ are elements in $\mathfrak{m}_{A}$, and $Q(x)$ is a non-degenerate quadratic form over A. Then there is an isomorphism

$$
R /(f(x)) \simeq R /\left(a^{\prime}+Q^{\prime}(x)\right)
$$

for some element $a^{\prime} \in \mathfrak{m}_{A}$ and some non-degenerate quadratic form $Q^{\prime}(x)$. 
Proof. This is [5], Chapter III, Proposition 2.4. We sketch the proof since it will be used to prove Proposition 2.6. We will find an element $b=\left(b_{1}, \ldots, b_{n}\right)$ with $b_{i} \in \mathfrak{m}_{A}$ such that the linear term of $f(x+b)$ vanishes and apply Lemma 2.7. We have

$$
f(x+b) \equiv f(b)+\sum_{i=1}^{n} a_{i} x_{i}+B(x, b)+L_{b}(x)+Q^{\prime}(x) \bmod I^{3},
$$

where $L_{b}(x)$ is a linear form with coefficients in the ideal $\left(b_{1}, \ldots, b_{n}\right)^{2}$ and $Q^{\prime}(x)$ is a quadratic form with $Q^{\prime}(x) \equiv Q(x) \bmod \mathfrak{m}_{A}$. Since $B$ is non-degenerate, there exists an element $b$ such that the linear term vanishes. This proves the lemma.

Remark 2.9. (1) If $a_{i} \in \mathfrak{m}_{A}^{r}$ for all $i$, where $r$ is a positive integer, then each component $b_{i} \in \mathfrak{m}_{A}^{r}$ and hence $a^{\prime}=f(b) \equiv a \bmod \mathfrak{m}_{A}^{2 r}$.

(2) The information $a^{\prime} \equiv a \bmod \mathfrak{m}_{A}^{3}$ in Proposition 2.6, which is not provided in [5], Chapter III, Proposition 2.4 (Lemma 2.8), is required in the proof of the main theorem.

Proof of Proposition 2.6. Condition (2.1) implies that

$$
f(x) \equiv a^{\prime}+\sum_{i=1}^{n} a_{i} x_{i}+Q^{\prime}(x) \bmod I^{3}
$$

such that $a^{\prime} \equiv a \bmod \mathfrak{m}_{A}^{3}$, each $a_{i} \in \mathfrak{m}_{A}^{2}$ and $Q^{\prime}(x) \equiv Q(x) \bmod \mathfrak{m}_{A}$. By Lemma 2.8 we have, for an appropriate element $b$,

$$
f(x+b) \equiv a^{\prime \prime}+Q^{\prime \prime}(x) \quad \bmod I^{3},
$$

where $a^{\prime \prime}$ is an element in $\mathfrak{m}_{A}$ such that $a^{\prime \prime} \equiv a^{\prime} \bmod \mathfrak{m}_{A}^{4}$ (since $a_{i} \in \mathfrak{m}_{A}^{2}$, see Remark 2.9) and $Q^{\prime \prime}(x) \equiv Q^{\prime}(x) \bmod \mathfrak{m}_{A}$. Therefore, by Lemma 2.7 .

$$
R /(f(x)) \simeq R /\left(a^{\prime \prime}+Q^{\prime \prime}(x)\right),
$$

where $Q^{\prime \prime}(x)$ is a non-degenerate quadratic form and $a^{\prime \prime}$ is an element in $\mathfrak{m}_{A}$ such that $a^{\prime \prime} \equiv a \bmod \mathfrak{m}_{A}^{3}$.

2.3. Singularities of $\mathbf{A}_{2, p, N}$. Let $x=(A, \lambda, \eta)$ be a point in $\Lambda \subset \mathcal{A}_{2, p, N}\left(\overline{\mathbb{F}}_{p}\right)$. Let $M$ be the associated quasi-polarized Dieudonné module. By Lemma 2.4, we choose a $W$-basis $X_{1}, X_{2}, Y_{1}, Y_{2}$ for $M$ as in the case (iib). It is easy to compute that the Hodge filtration Fil $\subset H_{1}^{D R}(A)$ is equal to $\left\langle Y_{1}, Y_{2}>_{k}\right.$, where $k=\overline{\mathbb{F}}_{p}$. Let $R^{\mathrm{u}}$ be the universal deformation ring of the quasi-polarized Dieudonné module $M$. It follows from Theorem 1.1 (1) that

$$
R^{\mathrm{u}}=R /(f), \quad R:=W\left[\left[t_{11}, t_{12}, t_{21}, t_{22}\right]\right]
$$

for some power series $f$. By the Grothendieck-Messing deformation theory, the universal deformation of $M$ over $R^{\mathrm{u}} / \mathrm{m}_{R^{\mathrm{u}}}^{p}$ is given by

$$
\widetilde{\text { Fil }}=<\widetilde{Y}_{1}, \widetilde{Y}_{2}>_{R^{\mathrm{u}} / \mathfrak{m}_{R^{\mathrm{u}}}^{p}} \subset H_{1}^{\text {crys }}\left(A /\left(R^{\mathrm{u}} / \mathfrak{m}_{R^{\mathrm{u}}}^{p}\right)\right),
$$

which is isotropic with respect to $\langle$,$\rangle , where$

$$
\tilde{Y}_{1}=Y_{1}+t_{11} X_{1}+t_{12} X_{2}, \quad \widetilde{Y}_{2}=Y_{2}+t_{21} X_{1}+t_{22} X_{2} .
$$

One computes the relation $\left\langle\widetilde{Y}_{1}, \widetilde{Y}_{2}\right\rangle=t_{11} t_{22}-t_{12} t_{21}+p=0$ in $R^{\mathrm{u}}$. This implies that

$$
f \equiv p+\left(t_{11} t_{22}-t_{12} t_{21}\right) \quad \bmod \mathfrak{m}_{R}^{p}
$$


This shows the following:

Lemma 2.10. If $x \in \Lambda$, then the moduli space $\mathcal{A}_{2, p, N}$ is not smooth at $x$.

Theorem 2.11. Assume $p>2$. Let $x=(A, \lambda, \eta)$ be a point in $\Lambda$. The formal completion of the local ring of $\mathbf{A}_{2, p, N}$ at $x$ is isomorphic to

$$
W\left(\overline{\mathbb{F}}_{p}\right)\left[\left[z_{11}, z_{12}, z_{21}, z_{22}\right]\right] /\left(p+z_{11} z_{22}-z_{12} z_{21}\right) \text {. }
$$

Proof. This follows immediately from Proposition 2.6 and (2.7).

\section{Singularities Using The LOCAL MODEL Method}

In this section we use the method of local models to determine the singularities of the moduli scheme $\mathbf{A}_{2, p . N}$, including the case where $p=2$. Our references are de Jong [3] and Rapoport-Zink [12].

3.1. Local model diagrams. Let $\Lambda_{1}:=\mathbb{Z}_{p}^{4}$ and $e_{1}, \ldots, e_{4}$ be the standard basis. Let $\psi$ be the alternating pairing on $\Lambda_{1}$ so that its representing matrix with respect to the standard basis is

$$
\left(\begin{array}{cc}
0 & I^{\prime} \\
-I^{\prime} & 0
\end{array}\right), \quad I^{\prime}=\left(\begin{array}{ll}
0 & p \\
1 & 0
\end{array}\right) .
$$

Let $\mathbf{M}^{\text {loc }}$ be the local model associated to the lattice $\Lambda_{1}$. This is the projective scheme over $\mathbb{Z}_{p}$ representing the following functor. For any $\mathbb{Z}_{p}$-scheme $S$, the set $\mathbf{M}^{\text {loc }}(S)$ of its $S$-valued points is the set of locally free $\mathcal{O}_{S}$-submodules $\mathcal{F} \subset \Lambda_{1} \otimes \mathcal{O}_{S}$, locally on $S$ direct summands of $\Lambda_{1} \otimes \mathcal{O}_{S}$, which are isotropic with respect to the pairing $\psi$.

Let $\widetilde{\mathbf{A}_{2, p, N}}$ be the moduli space over $\mathbb{Z}_{p}\left[\zeta_{N}\right]$ parameterizing equivalence classes of objects $(\underline{A}, \xi)$, where $\underline{A}$ is an object in $\mathbf{A}_{2, p, N}$ and

$$
\xi: H_{1}^{\mathrm{DR}}(A / S) \simeq \Lambda_{1} \otimes \mathcal{O}_{S}
$$

is an isomorphism which preserves the polarizations. Write $\widetilde{\mathcal{A}}_{2, p, N}$ for the reduction $\widetilde{\mathbf{A}}_{2, p, N} \otimes \overline{\mathbb{F}}_{p}$ modulo $p$.

Let $\mathcal{G}$ be the group scheme over $\mathbb{Z}_{p}$ representing the functor $S \mapsto \operatorname{Aut}\left(\Lambda_{1} \otimes \mathcal{O}_{S}, \psi\right)$. This group acts on the schemes $\widetilde{\mathbf{A}}_{2, p, N}$ and $\mathbf{M}^{\text {loc }}$ from the left. We have the following (local model) diagram:

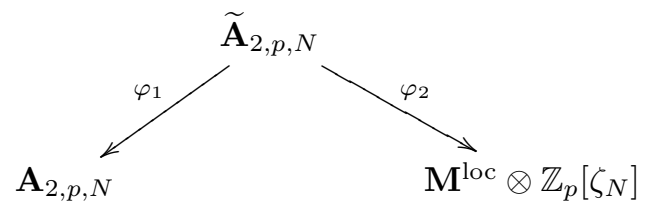

where

- $\varphi_{2}$ is the morphism that sends each object $(\underline{A} \bullet, \xi)$ to the image $\xi(\omega)$ of the Hodge submodule $\omega \subset H_{1}^{\mathrm{DR}}(A / S)$, and

- $\varphi_{1}$ is the morphism that forgets the trivialization $\xi$.

We know that

(i) the morphism $\varphi_{2}$ is $\mathcal{G}$-equivalent, surjective and smooth, and has the same relative dimension as $\varphi_{1}$ does, and

(ii) the morphism $\varphi_{1}: \widetilde{\mathbf{A}}_{2, p, N} \rightarrow \mathbf{A}_{2, p, N}$ is a $\mathcal{G}$-torsor. 
3.2. Singularities of $\mathbf{A}_{2, p, N}$. Let $x=(A, \lambda, \eta)$ be a point in $\Lambda \subset \mathcal{A}_{2, p, N}(k)$, where $k=\overline{\mathbb{F}}_{p}$. Let $M$ be the associated quasi-polarized Dieudonné module. By Lemma 2.4, we choose a $W$-basis $X_{1}, X_{2}, Y_{1}, Y_{2}$ for $M$ as in the case (iib). We choose a trivialization $\xi$ which sends $Y_{1}, X_{1}, X_{2}, Y_{2}$ to $e_{1}, e_{2}, e_{3}, e_{4}$, respectively. This defines a point $y \in \widetilde{\mathcal{A}}_{2, p, N}$ mapping to $x$. We put $z=\varphi_{2}(y) \in \mathbf{M}^{\text {loc }}\left(\overline{\mathbb{F}}_{p}\right)$, which is given by

$$
<e_{1}, e_{4}>_{k} \subset \Lambda_{1} \otimes k .
$$

Around the point $z$ we choose the same coordinates as in Subsection 2.3 so that an affine open $\operatorname{chart} \mathcal{U}$ is

$$
\operatorname{Spec} W\left[t_{11}^{\prime}, t_{12}^{\prime}, t_{21}^{\prime}, t_{22}^{\prime}\right] /\left(p+t_{11}^{\prime} t_{22}^{\prime}-t_{12}^{\prime} t_{21}^{\prime}\right) .
$$

Let $R_{x}$ (resp. $R_{y}$ and $R_{z}$ ) be the completion of the local ring of $\mathcal{A}_{2, p, N}$ at $x$ (resp. of $\widetilde{\mathcal{A}}_{2, p, N}$ at $y$ and of $\mathbf{M}^{\text {loc }}$ at $z$ ). We have

$$
R_{x}\left[\left[t_{1}, t_{2}, t_{3}\right]\right] \simeq R_{y} \simeq R_{z}\left[\left[s_{1}, s_{2}, s_{3}\right]\right]
$$

and

$$
R_{z}=W\left[\left[t_{11}^{\prime}, t_{12}^{\prime}, t_{21}^{\prime}, t_{22}^{\prime}\right]\right] /\left(p+t_{11}^{\prime} t_{22}^{\prime}-t_{12}^{\prime} t_{21}^{\prime}\right) .
$$

By 3], Lemma 4.7, there is an isomorphism

$$
R_{x} \simeq R_{z}=W\left[\left[t_{11}^{\prime}, t_{12}^{\prime}, t_{21}^{\prime}, t_{22}^{\prime}\right]\right] /\left(p+t_{11}^{\prime} t_{22}^{\prime}-t_{12}^{\prime} t_{21}^{\prime}\right) .
$$

This shows Theorem 1.3.

\section{Remark 3.1.}

(1) Using local models is a very effective method for computing local moduli spaces. However, one can apply it only in the special cases when the kernels of polarizations are annihilated by $[p]$.

(2) The isomorphism (3.2) is obtained by a successive approximation. Therefore, we were not able to tell whether the coordinates $t_{i j}^{\prime}$ are global ones near the point $x$. Lemma 4.1 shows some evidence that they might not be the global coordinates near the singularities.

\section{Some Fine information from the Cartier theory}

As experts all know that there is another important tool for computing local moduli spaces - using the Cartier theory, we discuss our situation with this method. First of all, the method works exclusively only in characteristic $p$; namely, the best information we can get is the local moduli spaces modulo $p$. In 9 Norman established the theoretic background for an algorithm for computing local moduli spaces. In principle the algorithm can only compute the coordinate ring of the universal deformation space modulo a specific power of the maximal ideal. However, since the singularity is determined by its sufficiently well approximation, one would be able to determine the singularity eventually. Norman gave two examples in 9, and the first example is exactly the case of $\mathcal{A}_{2, p, N}$ at points in $\Lambda$.

Let $M$ be the Dieudonné module associated to a point $x$ in $\Lambda$. We can choose a basis $\left\{e_{i}\right\}$ for $M$ so that (cf. (iib) $) \sqrt{1}$

$$
\begin{array}{ll}
F e_{1}=e_{3}, & F e_{2}=e_{4}, \\
e_{3}=V e_{1}, & e_{4}=V e_{2},
\end{array}
$$

\footnotetext{
${ }^{1}$ We use the same notation as in [9] except that $t_{i j}$ and $T_{i j}$ are switched. This choice may make it easier for the reader who is interested in reading the calculation in [9]. The correspondence with the basis in (iib) is $e_{1}, e_{2}, e_{3}, e_{4}$ corresponding to $X_{1}, X_{2}, Y_{1}, Y_{2}$, respectively.
} 
and that the quasi-polarization $P$ from $M$ to its dual $M^{t}$ is given by

$$
P\left(e_{1}\right)=-f_{2}, \quad P\left(e_{2}\right)=f_{1},
$$

where $\left\{f_{i}\right\}$ is the dual basis for $M^{t}$. It is easy to see that

$$
\begin{aligned}
& f_{1}=V f_{3}, \quad f_{2}=V f_{4}, \\
& F f_{3}=f_{1}, \quad F f_{4}=f_{2},
\end{aligned}
$$

and that

$$
P\left(e_{3}\right)=-p f_{4}, \quad P\left(e_{4}\right)=p f_{3} .
$$

Using the theory of displays, one constructs the equi-characteristic universal deformation $\widetilde{M}$ over the universal deformation ring $R_{0}=k\left[\left[t_{11}, t_{12}, t_{21}, t_{22}\right]\right]$, which is generated by $\left\{e_{i}\right\}$ over the Cartier ring $\operatorname{Cart}_{p}\left(R_{0}\right)$ (the ring $A_{R_{0}}$ in [9]) with the following relations:

$$
\begin{aligned}
F e_{1} & =e_{3}+T_{11} e_{1}+T_{12} e_{2}, \\
F e_{2} & =e_{4}+T_{21} e_{1}+T_{22} e_{2}, \\
e_{3} & =V e_{1}, \\
e_{4} & =V e_{2},
\end{aligned}
$$

where $T_{i j}=\left[t_{i j}\right]$ is the Teichmüller lifting of $t_{i j}$. Let $f \in R_{0}$ be the defining equation (up to a unit) of the maximal closed formal subscheme over which the quasi-polarization $P$ extends. Norman showed that (when $p>2$ ):

$$
f=t_{11} t_{22}-t_{12} t_{21}+r(t), \quad \text { for some } r(t) \in\left(t_{i j}\right)^{p} .
$$

Since the singularity of $R_{0} /(f)$ is determined by its leading term, one computes the local moduli space in characteristic $p$. However, if one would like to know the non-ordinary locus of the local moduli space, then the calculation of the remaining term $r(t)$ is required; see the following lemma.

Lemma 4.1. The non-ordinary locus of the local moduli space $\operatorname{Spec} R_{0} /(f)$ is $\operatorname{Spec} R_{0} /\left(t_{11} t_{22}-t_{12} t_{21}, f\right)=\operatorname{Spec} R_{0} /\left(t_{11} t_{22}-t_{12} t_{21}, r(t)\right)$.

Proof. The Frobenius map on the tangent space $\widetilde{M} / V \widetilde{M}$ of the universal deformation $\widetilde{M}$ is given by

$$
F e_{1}=t_{11} e_{1}+t_{12} e_{2}, \quad F e_{2}=t_{21} e_{1}+t_{22} e_{2} .
$$

Therefore, the defining equation of the non-ordinary locus is $t_{11} t_{22}-t_{12} t_{21}$.

\section{ACKNOWLEDGMENTS}

The author thanks J. Tilouine for his interest in the work. The research was partially supported by the grants NSC 97-2115-M-001-015-MY3 and AS-98-CDAM01. The author also thanks the referee for helpful comments that improved the exposition.

\section{REFERENCES}

[1] J. D. Achter, Hilbert-Siegel moduli spaces in positive characteristic. Rocky Mountain J. Math. 33 (2003), 1-25. MR.1994479(2004e:14071)

[2] A. J. de Jong, The moduli spaces of polarized abelian varieties. Math. Ann. 295 (1993), 485-503. MR1204833 (94g:14024)

[3] A. J. de Jong, The moduli spaces of principally polarized abelian varieties with $\Gamma_{0}(p)$-level structure. J. Algebraic Geom. 2 (1993), 667-688. MR1227472(94d:14042) 
[4] Groupes de monodromie en géométrie algébrique. II. Séminaire de Géométrie Algébrique du Bois-Marie 1967-1969 (SGA 7 II). Dirigé par P. Deligne et N. Katz. Lecture Notes in Math., vol. 340, Springer-Verlag, 1973. MR0354657 (50:7135)

[5] E. Freitag and R. Kiehl, Etale cohomology and the Weil conjecture. Ergebnisse der Mathematik und ihrer Grenzgebiete (3), 13, Springer-Verlag, Berlin, 1988. MR.926276 (89f:14017)

[6] A. Grothendieck, Groupes de Barsotti-Tate et Cristaux de Dieudonné. Les Presses de l'Université de Montréal, 1974. MR0417192 (54:5250)

[7] N. Koblitz, $p$-adic variant of the zeta-function of families of varieties defined over finite fields. Compositio Math. 31 (1975), 119-218. MR0414557(54:2658)

[8] W. Messing, The crystals associated to Barsotti-Tate groups: with applications to abelian schemes. Lecture Notes in Math., 264, Springer-Verlag, 1972. MR0347836 (50:337)

[9] P. Norman, An algorithm for computing moduli of abelian varieties. Ann. of Math. (2) 101 (1975), 499-509. MR0389928 (52:10757)

[10] P. Norman and F. Oort, Moduli of abelian varieties, Ann. of Math. (2) 112 (1980), 413-439. MR.595202 (82h:14026)

[11] F. Oort, Finite group schemes, local moduli for abelian varieties, and lifting problems. Algebraic Geometry (Oslo) 223-254 (1972). MR0301026 (46:186)

[12] M. Rapoport and Th. Zink, Period spaces for p-divisible groups. Ann. Math. Studies 141, Princeton Univ. Press, 1996. MR.1393439 (97f:14023)

[13] J. Tilouine, Siegel Varieties and $p$-Adic Siegel Modular Forms. Doc. Math. Extra Volume: John H. Coates' Sixtieth Birthday (2006), 781-817. MR.2290605 (2007k:11067)

[14] C.-F. Yu, Lifting abelian varieties with additional structures. Math. Z. 242 (2002), 427-441. MR.1985459 (2004m:14095)

[15] C.-F. Yu, The supersingular loci and mass formulas on Siegel modular varieties. Doc. Math. 11 (2006), 449-468. MR2288077 (2007m:11081)

Institute of Mathematics, Academia Sinica, Astronomy-Mathematics Building, 6th Floor, No. 1, Roosevelt Road, Sec. 4, Taipei, Taiwan - And - NCTS (Taipei Office)

E-mail address: chiafu@math.sinica.edu.tw 